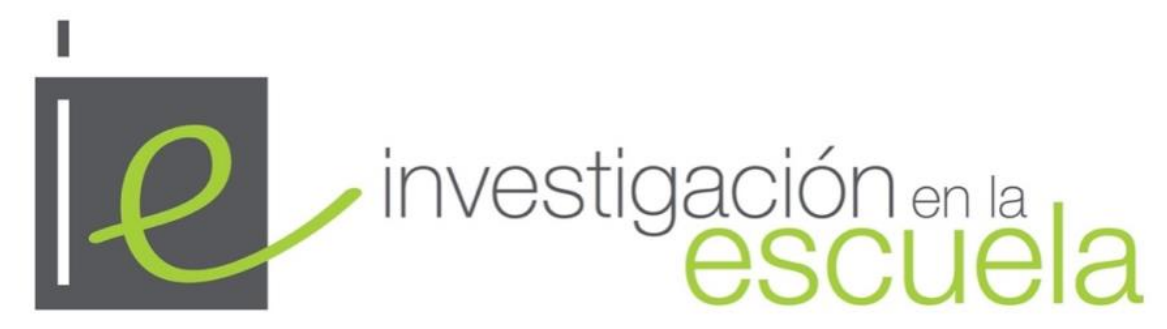

Revista de Investigación e Innovación Educativa nº 105, 2021 | e-ISSN 2443-9991

\title{
Innovar para transformar la sociedad. Una revisión desde el ámbito universitario
}

Innovating for transforming the society. A review from the university scenario

D. Víctor Valdés Sánchez, es investigador en la Universidad de Extremadura (España)·victorvs@unex.es· Orcid https://orcid.org/0000-0003-0478-9814

Dra. Prudencia Gutiérrez Esteban, es Profesora Titular del Departamento de Ciencias de la Educación de la Facultad de Educación en la Universidad de Extremadura (España) · pruden@unex.es · Orcid https://orcid.org/0000-00031-5328-5319

Cómo citar este artículo

Valdés Sánchez, V. y Gutiérrez Esteban, P. (2021). Innovar para transformar la sociedad. Una revisión desde el ámbito universitario. Investigación en la Escuela, 105, 66-76. doi: https://doi.org/10.12795/IE.2021.i105.06

Resumen. La innovación educativa es considerada como una herramienta imprescindible para resolver los principales retos del sistema educativo y transformar las prácticas que tienen lugar. Así, las instituciones educativas y especialmente, las universitarias, juegan un papel crucial y deben hacer un esfuerzo por mejorar y dirigir sus energías a alcanzar una sociedad más justa, solidaria, intercultural y diversa, ofreciendo una mayor responsabilidad e interacción social. Además, la puesta en práctica de una innovación social, con carácter abierto, contribuye a la identificación de problemas y la búsqueda de soluciones desde la Universidad y a la generación de una comunidad educativa duradera. El presente artículo realiza una revisión teórica sobre el papel de las universidades, en concreto de las Facultades de Formación del Profesorado, en la transformación educativa y social. Entre los principales hallazgos, se precisa la necesidad de diseñar una formación inicial del profesorado creativa y de vanguardia, capaz de responder a los cambios sociales, económicos y políticos, de manera que capacite al futuro profesorado como agentes sociales a través de procesos reflexivos que posibiliten la construcción y ensamblaje de las características necesarias para formar docentes contemporáneos, capaces de vencer el anacronismo que impera en la actualidad y avanzar hacia la transformación educativa y social.

Abstract. Educational innovation is considered essential and as a tool for change in order to solve the main challenges of the educational system and transform the practices that take place. Thus, educational institutions and specially, Higher Education Institutions play a crucial role and an effort must be made with the aim of improving their capacity to lead these strengths towards a more just, supportive, intercultural and diverse society. In addition, the implementation of social innovation, with an open character, contributes to the identification of problems and the search for solutions from the University and to the generation of a lasting educational community. This article carries out a theoretical review of the role of universities, specifically of Teacher Training Faculties, in educational and social transformation. Among the main findings, the design of a creative and avant-garde initial teacher training, capable of responding to social, economic and political changes, is required, in order to train future teachers as social agents through reflective processes that enable the construction and assembly of the necessary characteristics to train contemporary teachers, capable of overcoming the anachronism that prevails today and move towards educational and social transformation.

Palabras clave $\cdot$ Keywords

Innovación pedagógica, universidad, transformación educativa, interacción social, cambio social. Educational innovations, university, educational transformations, social interaction, social change. 


\section{Introducción}

Desde una perspectiva de futuro, parece inequívoco que la Educación requiere una mayor integración social. Los sistemas educativos, deben abrirse para desarrollar en la ciudadanía conocimientos más sociales mediante una participación activa en los procesos de aprendizaje (Carbonell, 2002). Desde las posiciones de Habermas (1982) y Barraza et al. (2013), se trata de otorgar a la Educación un carácter más crítico, superar el intelectualismo puro para pasar a la práctica, conectando con los problemas cotidianos educando en y para esas realidades. En palabras de Roa (2017):

la Educación ha dejado de entenderse y postularse como un tipo de saber filosófico que repercute en el modo de ser de quien la piensa y la vivencia (...) para situarse en el campo de la economía política, (...) que supedita su objetivo a las condiciones de la economía y que, por tanto, aparece desprovista de su sentido esencialmente humano, para quedar convertida en objeto de consumo (p.110).

Estudios en paralelo, como el de Palacios-Acosta y Sainz-Ucros (2019), alertan de que los centros educativos no pueden relegarse únicamente al desarrollo intelectual y tampoco deben perderse dentro de las corrientes que estandarizan la Educación y olvidan la atención a las particularidades del alumnado. Proponen, por consiguiente, la concepción de un quehacer afectivo-pedagógico que engloba todo aquello que trasciende en Educación. De todo ello, desde la administración educativa deben responsabilizarse impulsando políticas que promuevan el sentido social y humano de la Educación.

La realización de estos cambios en Educación sostiene Huertas (2013), supondría desde una visión totalmente ampliada, la transformación de la sociedad hacia una comunidad más humana, donde se realice una puesta en valor de lo social, de los cuidados y, en definitiva, de apostar por colocar la vida en el centro. En Educación, poner la vida en el centro requiere alejarla de la violencia, el miedo, la exclusión, el machismo o la jerarquía, para empezar a colocar las relaciones respetuosas en el centro de la vida social, poner en valor la importancia de los cuidados y el buen vivir (Tomé, 2019).

\subsection{La innovación social como herramienta de cambio en el sistema educativo}

La educación comunitaria se lleva a cabo mediante procesos de aprendizaje social, lo que supone realizar una serie de cambios en el sistema educativo, como son: la potenciación de valores como la responsabilidad o la solidaridad; la puesta en práctica de metodologías de aprendizaje colaborativo donde se apliquen técnicas para la resolución conjunta de problemas; la inclusión de todos los agentes que forman parte de la comunidad educativa o la apertura de las escuelas para ampliar la concepción de espacios educativos (Cieza, 2006).

Así, se comienza a hablar de la aplicación de la innovación social, como herramienta de cambio, pues como Blanco y Bonell (2019) defienden, la innovación debe servir para dar respuesta a los retos del futuro sabiendo que el cambio no es solo tecnológico, el conocimiento no es solo científico y hay muchos más valores que el económico.

Echevarría (2008) habla de innovación social para referirse a valores como el bienestar, la inclusión social, la solidaridad, la participación social o la calidad medioambiental entre otros. En síntesis, este concepto podría definirse, como apunta Cotte y Gámez (2012), como una forma novedosa de dar respuesta a necesidades y problemas sociales. Sureda (2017) establece los retos de la innovación social para garantizar su éxito en los siguientes puntos:

- Propiciar un mayor acercamiento a la sociedad.

- Asegurar nuevos espacios de participación e interacción.

- Trabajar colaborativamente.

- Generar impacto.

- Buscar alternativas para la obtención de recursos.

- Plantear la innovación de manera abierta.

- Aprovechar las oportunidades de las tecnologías.

Trabajos como el de Acosta (2018), apuestan por la innovación social educativa como un proceso de autogestión y autorregulación, capaz de generar una comunidad educativa duradera. Aunque en el ámbito educativo aún no es muy conocida y queda mucho camino para que sea interiorizada e institucionalizada, Schröder \& Krüger (2019) la consideran totalmente imprescindible para ser capaces de solucionar los principales problemas del sistema educativo. Sugieren, además, la puesta en práctica de una innovación social con carácter abierto que sea capaz de involucrar a toda la comunidad en los proyectos de innovación. Sales et al. (2019) señalan la participación de la comunidad educativa como uno de los factores clave para 
alcanzar esa transformación educativa. De esta forma, todos los agentes que conforman la comunidad educativa deben estar incluidos en procesos participativos, creativos y colaborativos para encontrar y diseñar conjuntamente nuevos modelos educativos que se adapten al contexto de intervención. En este sentido, Novoa y Camacho (2017), vinculan la innovación en el ámbito educativo con los procesos comunitarios, pues es en ellos donde se puede identificar problemas y buscar soluciones provocando la puesta en marcha de proyectos de innovación.

Como consecuencia, se generan actuaciones colectivas que son capaces de perdurar en el tiempo más allá de las actuaciones que se realizan de manera aislada en la soledad del aula. De la misma forma, pero de manera inversa, Roa (2017) considera que los procesos de innovación posibilitan la construcción colectiva de nuevos hábitos sociales. A modo de conclusión, en palabras de Blas García (2018):

Ninguna innovación educativa debería considerarse tal a menos que sirviese para enseñar a ser, para capacitar el afrontamiento y resiliencia ante las dificultades personales y sociales, para dotar de competencias dirigidas a mejorar las relaciones humanas. En definitiva, para construir un mundo mejor y más humano (p.10).

\subsection{La Universidad en la transformación educativa y social}

Con independencia del nivel educativo al que nos refiramos, Palacios-Acosta y Sainz-Ucros (2019) sostienen que en todos estos niveles existe una responsabilidad social latente que vincula el currículum educativo con las necesidades del contexto.

En la evolución histórica de las universidades, Grosso (2018) argumenta que se ha reducido, en gran medida, dicha interacción con las fuerzas sociales y culturales del cambio, con los agentes locales y, en definitiva, se ha perdido parte de la singularidad de cada Universidad. De esta forma, se extiende una visión homogeneizadora de la institución, conforme a estándares internacionales que la orientan más hacia la interactuación con actores empresariales, nacionales e internacionales, perdiendo de esta forma su capacidad, en muchos casos, para dar respuesta a la transformación hacia una sociedad más justa, solidaria, intercultural y diversa.

La Responsabilidad Social (RS), como destaca Domínguez Pachón (2009), es una forma de solicitar a las organizaciones que den respuesta a las implicaciones de su actividad en el mundo. El sector empresarial ha desarrollado políticas de RS en los últimos años promovidas en gran medida por la reivindicación de los derechos humanos, la transparencia o la protección del medio ambiente, entre otras cuestiones, que ha demandado la ciudadanía en aras de un mundo mucho más justo y ético. Este movimiento llega a las universidades como "Responsabilidad Social Universitaria" (RSU), es decir, la capacidad de la institución de desarrollar y difundir principios y valores sociales a través de sus principales actividades.

Profundizando un poco más en la RSU, la Universidad además del papel que realiza en la docencia, la investigación y la preparación técnica para el ejercicio de una profesión, no debe olvidar su responsabilidad con la transformación social, debiendo actuar ante los problemas locales, regionales, nacionales y universales y abogando por un mundo más sostenible, igualitario y justo. En cambio, parece que la carga burocrática junto con las estáticas estructuras organizativas o el excesivo control que sufren, reduce su autonomía y merma su capacidad innovadora, impidiendo en gran medida dedicar esfuerzos a formar una ciudadanía crítica, ética y comprometida (Lampert, 2003).

Leite y Beltrán (2012) sintetizan muy bien esta idea, demandando que la Universidad no solo debe explicar la realidad y aplicar conocimiento en ella, sino que debe implicarse también en esa realidad. De manera que debe mutar desde su concepción primigenia de una organización que enseña, a una estructura de organización que aprende. Así, Garnica y Franco (2020) sugieren que la Universidad debe acoger la innovación de forma integral en la docencia, la investigación, la infraestructura y la administración.

Vinculando la RSU con la Innovación Social desarrollada en el apartado anterior, Villa (2014) propone un Sistema de Innovación Social Universitario Responsable (ISUR) que se sustenta sobre seis dimensiones, estrechamente relacionadas con los principales ámbitos en los que interviene la Universidad (docencia, gestión, investigación y extensión universitaria) (Torres Pedreros, 2016). A continuación, se abordan en profundidad cada una de ellas:

- Dimensión diseño curricular y pedagógico: Se trata de la inclusión de actividades académicas con un objetivo social, como por ejemplo la formación de valores o el desarrollo de competencias para la ciudadanía.

- Dimensión organizativa: Implica el funcionamiento participativo de la Universidad y su cultura de organización. 
- Dimensión investigación, desarrollo e innovación: Se trata de poner la investigación que se desarrolla dentro de las universidades al servicio de los problemas y necesidades que se identifican en el entorno.

- Dimensión ambiental: Trata de integrar en la docencia, la investigación y la política de la Universidad prácticas ambientalmente responsables.

- Dimensión relación con el entorno: Supone la transferencia de conocimiento de la Universidad a la sociedad. En otras palabras, cosiste en medir el impacto de la actividad universitaria sobre el contexto.

- Dimensión internacionalización: Consiste, por una parte, en establecer redes de intercambio de conocimiento y cooperación entre universidades y, por otra parte, la formación de profesionales que hayan integrado una perspectiva glocal (global+local), que les permita comprender las repercusiones globales de las prácticas locales.

En relación con las dimensiones abordadas en el Sistema de Innovación Social Universitario, es necesario que las universidades apliquen, como sugieren Navas y Romero (2016), diferentes políticas que les permitan materializar cada una de las dimensiones. De esta forma se recomienda establecer una estrategia basada en políticas de gestión y calidad institucional, gestión medioambiental responsable, formación académica socialmente responsable, políticas de egresados que favorezcan la construcción de perfiles de profesionales solidarias y comprometidas con el entorno y políticas de investigación útil y gestión social del conocimiento.

Ante estas propuestas, uno de los debates que permanece abierto es el futuro de las universidades, como apunta Villa (2020), y más concretamente el de las universidades públicas. Entre las cuestiones que se discuten, se encuentra la relación entre Universidad y Sociedad, que se está abordando a lo largo de este apartado y sobre la que Serna Alcántara (2007) enumera una serie de circunstancias que evidencian la necesidad de mejorar la labor de la extensión social universitaria.

- La reducción de la labor de extensión en la Universidad.

- La limitación de la labor social a compromisos individuales.

- La disminución de la influencia de la Universidad en la resolución de problemas sociales.

- El desaprovechamiento de recursos y capital humano.

- La falta de formación, concienciación y motivación del alumnado para la transformación social.

Desde otra perspectiva, pero en la misma línea, la UNESCO (1998) o más recientemente, Alférez y Comas (2015) y Cisneros Quintanilla y Mendoza Bravo (2018) trazan, mediante una serie de propuestas, el camino que debería seguir la Universidad para dar respuesta a su responsabilidad social y la vinculación con la sociedad que se espera de esta institución. Se trata de:

- Vincular la sociedad como una de las funciones de la Universidad.

- Diseñar un proceso educativo/formativo, transformador e integrador.

- Generar una relación bidireccional entre Universidad-Sociedad.

- Convertirse y afianzarse como un espacio de aprendizaje, reflexión y acción social interdisciplinaria.

- Transferir del trabajo educativo universitario a la sociedad.

- Crear espacios de prácticas sociales compartidas y colaborativas para comprender e intervenir de manera creativa e innovadora en las situaciones identificadas con problemas sociales.

De forma más concreta y centrándose en el alumnado, García Ramos et al. (2016) profundizan sobre el constructo "responsabilidad social del alumnado universitario" acotándolo a las dimensiones de pensamiento crítico, empatía y capacidad de compromiso. De esta forma, es totalmente necesario formar al alumnado universitario en valores, en la capacidad de escucha, en el compromiso para con las demás personas y en la capacidad de diálogo. Se trata de aportar los recursos necesarios para que puedan ser profesionales responsables en su campo de especialización.

\section{Metodología}

Para la revisión teórica que se lleva a cabo en este artículo, se ha realizado una selección de la bibliografía especializada existente. En concreto, se han consultado más de 60 textos diferentes entre documentos académicos y legislativos. La selección de estos documentos se ha realizado a partir de la búsqueda de bibliografía especializada, para la cual se han utilizado diferentes descriptores tales como: Innovación Social (Social Innovation), Responsabilidad Social Universitaria (University Social Responsibility), Formación Inicial del Profesorado (Initial Teacher Training), en diferentes bases de datos, tales como Scopus, Dialnet y Web of Science (WOS).

Tras la selección de los textos, por su idoneidad con el objeto de estudio, se procedió a realizar una lectura en profundidad de cada uno de ellos y así, extraer las principales ideas, resultados y conclusiones que contribuían a comprender el estado de la cuestión. Tras un proceso de lectura y análisis, se realizó la 
comparación de los diferentes textos, para elaborar una narrativa que permitiese comprender el papel de las universidades, en concreto de las Facultades de Formación del Profesorado, en la transformación educativa y social como base para futuros estudios e investigaciones.

Una de las principales limitaciones de este estudio, es que se trata de un análisis exploratorio, realizado a partir de una serie de publicaciones seleccionadas por su relevancia e interés para el análisis que se lleva a cabo. En este sentido, a pesar de que no se hace una revisión sistemática de la bibliografía especializada, se considera que el número de documentos analizados, es suficiente para reflexionar sobre el papel de las universidades en la transformación social.

Tras la revisión de la literatura identificada como pertinente, se encuentran algunos indicadores y marcos conceptuales comunes sobre la innovación social y la educación, coincidentes con los hallazgos revelados por Surikova et al. (2015), los cuales se describen a continuación.

\section{La Educación y la innovación social}

Actualmente, los sistemas educativos están estancados en modelos clásicos customizados con nuevos materiales didácticos y el empleo masivo de las tecnologías. En esta misma línea, se habla de las pedagogías emergentes como un modelo de innovación abierta al usuario (Adell y Castañeda, 2012). Para Gros (2015), las pedagogías emergentes han hecho desaparecer lo que hasta ahora eran los muros del conocimiento. "Dado que todos los componentes de las pedagogías emergentes (...) están en constante evolución, los profesionales de la Educación precisan estrategias de constante adaptación y cambio para entender cómo estos componentes interaccionan y modifican sus propias prácticas" (Gros, 2015, p. 64). Tanto es así que Adell y Castañeda (2012) valoran que estos nuevos enfoques no han sido ni lo suficientemente comprendidos ni estudiados.

En cambio, no parece que sea éste el modo de transformar la Educación. Desde otros sectores de la sociedad que también están experimentando una transformación similar, consideran clave la puesta en marcha de un cambio de paradigma. En el caso de la Educación, se trata de un nuevo paradigma educativo que sea capaz de dar respuesta a las necesidades y problemas del futuro (Aguerrondo, 1999). Fullan (2002) propone este cambio de paradigma educativo, estructurado en ocho principios fundamentales sintetizados por Turrado (2015):

- Las cuestiones importantes en el sistema educativo no pueden aplicarse mediante la imposición, es necesario establecer canales de diálogo con los agentes educativos.

- Las teorías de la Educación se pueden complementar con las teorías generadas para entender los procesos de cambio que imperan en nuestra sociedad.

- La diversidad y el conflicto deben incluirse dentro del sistema pues son parte del contexto educativo.

- La gestión de la incertidumbre es una de las competencias clave que se debe incorporar de manera urgente para saber desenvolverse en un futuro cada vez más incierto.

- La inteligencia emocional supone una herramienta indispensable para hacer frente a muchas de las denominadas "enfermedades del progreso", como la depresión o la ansiedad.

- Debe buscarse el equilibrio entre el individualismo y el colectivismo. En una sociedad cada vez más egoísta, sigue siendo necesario garantizar una Educación personalizada, pero tiene necesariamente que poseer una esencia comunitaria.

- Resulta necesario utilizar el grado de conectividad que nos ofrecen las tecnologías para el establecimiento de redes colaborativas para mantenernos actualizados, pero siempre manteniendo un espíritu crítico que nos permita analizar la ingente cantidad de información que se tiene actualmente al alcance de la mano.

- Hay que generar nuevo conocimiento y nuevas teorías que se adapten a cada realidad, pues la gran diversidad presente en la sociedad dificulta el establecimiento de explicaciones universales que sean aplicables a cualquier contexto.

Al mismo tiempo, una interesante propuesta es la que realizan Surikova et al. (2015), al identificar un modelo conceptual que aborda la interacción entre la Educación y la innovación social, toda vez que avanzan en la construcción de un nuevo modelo conceptual sobre el triple rol de la educación en la promoción de la innovación social, bajo tres perspectivas, a saber, las necesidades sociales (Educación percibida como necesidad social y fuente de resolución de problemas), los retos sociales (Educación como reto social y fuente de recursos humanos para el empleo en la innovación social) y los cambios sistémicos (Educación como indicador de calidad de vida y fuente de nuevas oportunidades y enfoques a identificar). En este 
modelo se identifican temas comunes, recursos humanos y nuevas oportunidades que deben ser concebidas, investigadas y desarrolladas desde una perspectiva global (Figura $\mathrm{n}^{\circ} 1$ ).

\section{Figura 1}

Modelo conceptual de interacción entre innovación social y educación (por Surikova et al., 2015, p. 237).

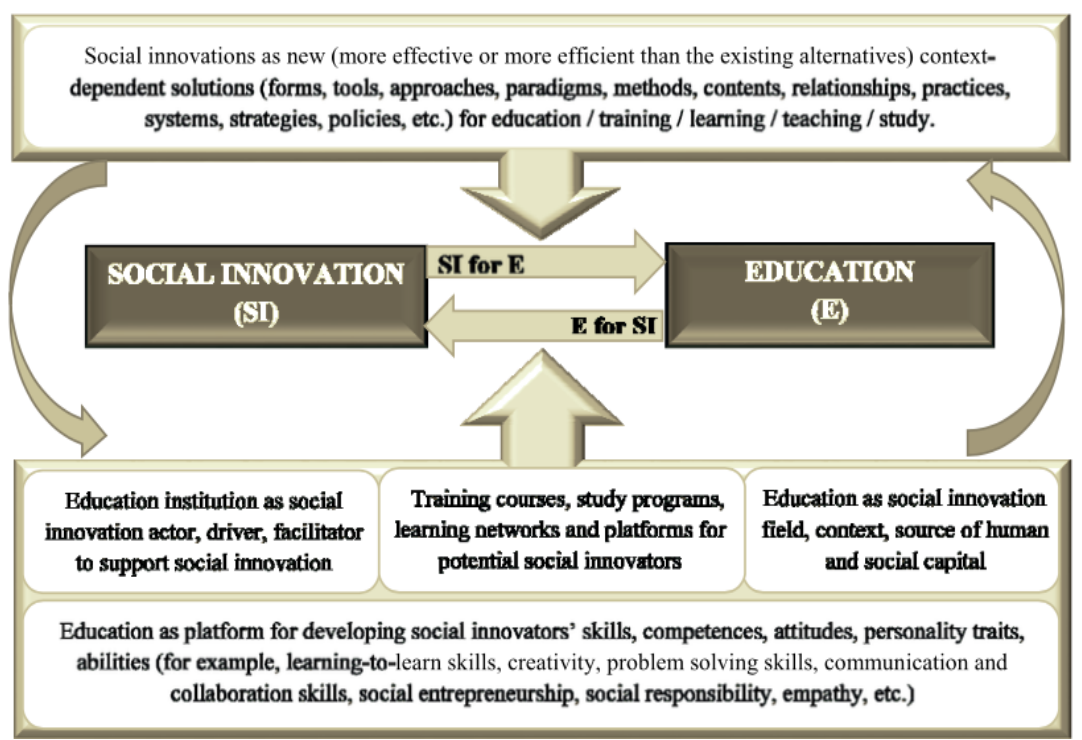

Esto supone como prevé Sancho Gil (2010), involucrar al alumnado en proyectos personales y sociales, fomentar la cohesión social, actualizar los conocimientos de profesorado y la administración y reforzar las relaciones entre el sistema educativo y el sistema social. En el camino hacia el cambio educativo con un mayor calado social, Álvarez Álvarez (2015) defiende la superación de la innovación por parte de la comunidad educativa, comenzando a hablar de transformación educativa como búsqueda para dar respuesta a esta nueva estructura de sociedad.

Tanto es así que Sancho Gil y Correa Gorospe (2010) reflexionan sobre una serie de medidas que son necesarias aplicar en la práctica educativa si se quiere favorecer la transformación educativa. Se trata de concebir al alumnado no como una tabula rasa, sino como personas activas en los procesos de aprendizaje, teniendo en cuenta también su realidad fuera del centro educativo y todo lo que esto implica. Se requiere cambiar también la manera de entender los saberes, que han dejado de ser una constante para necesitar una revisión y actualización permanente (Contreras y Pérez de Lara, 2010). Por último, creen necesario interiorizar las implicaciones de vivir en un mundo cada vez más digital y repensar qué significa la "alfabetización" en la sociedad actual.

\subsection{La realidad de la formación inicial del profesorado}

Para ello, se revela como más necesario que nunca recabar la visión del profesorado y alumnado universitario sobre la cuestión de la formación inicial, voces que ya comenzamos a estudiar hace años y que nos permitieron profundizar en interesantes hallazgos acerca del papel de las facultades de Educación en la formación inicial de este colectivo (Valdés y Gutiérrez-Esteban, 2016). En ese sentido, la principal preocupación percibida era la dificultad que supone desarrollar estrategias metodológicas en aulas masificadas. Esto implica para el profesorado universitario un escollo difícil de salvar, que hace que desarrollen su trabajo en condiciones no exentas de dificultades. Ligada a esta masificación, también se mencionaba la motivación que posee el alumnado que comienza la formación inicial en los grados de Educación.

La situación, de quienes serán docentes, es diversa a la hora de comenzar los estudios, dado que se percibe una falta de vocación entre este grupo, que conformará el futuro cuerpo docente. En cambio, elementos tales como la ampliación un año más del plan de estudios del Grado de Maestro, como consecuencia de los cambios acaecidos tras la implantación del EEES, fue valorado muy positivamente junto con el considerable incremento del Practicum, tanto en número de créditos como en tiempo de permanencia en las escuelas, como también recoge el anterior estudio de Gutiérrez-Esteban (2011). Lo que deja entrever el alejamiento de la teoría-práctica hacia un enfoque más práctico-práctico (Díaz Quero, 2004). Además, más allá de las prácticas, se demanda una mayor relación entre Universidad y escuela. Respecto a 
la opinión del alumnado universitario, se detectó la demanda de un cambio más profundo en la formación inicial, incidiendo en la necesidad de que se les capacite en competencias más que en contenidos, los cuales ya se han trabajado en niveles anteriores, o bien, están a su alcance a través de las tecnologías.

Así, se desprende la necesidad de mejorar la formación para que pueda hacer frente a las nuevas habilidades y competencias del siglo XXI. En este sentido, Del Rosal et al. (2018), abogan por la incorporación y mejora de la formación en materia de inteligencia emocional, con el fin de dotar al profesorado de competencias que les permitan hacer frente a las nuevas situaciones que se producen en los contextos de aprendizaje actuales. Al mismo tiempo, Hernández-Rincón et al. (2012) destacan la importancia de la acreditación de la expresión oral y escrita, la madurez personal y la alfabetización digital entre otras habilidades en la formación inicial del profesorado, llegando a plantear incluso cursos cero o de nivelación para la preparación de estas habilidades que consideran imprescindibles para encaminar la formación de docentes hacia las necesidades del futuro. También desde esta línea de pensamiento, Gutiérrez-Esteban y Becerra-Traver (2014) y Valverde Berrocoso (2015), proponen la inclusión de herramientas y metodologías que permitan al futuro profesorado adquirir las competencias necesarias para desenvolverse en la sociedad digital.

Desde otra perspectiva, Moreno-Crespo y Moreno-Fernández (2015), al estudiar la formación inicial del profesorado en Sevilla y Extremadura, revelan que el alumnado de estas titulaciones no se siente capacitado para tratar problemas relevantes en el aula, como son los problemas socio-medioambientales. Por su parte, Borrero y Blázquez (2018), desde la defensa de una mejor formación en materia de interculturalidad, defienden un cambio del modelo pedagógico sustentado en la implicación y en el trabajo colaborativo entre el profesorado.

Por otra parte, si tomamos como referencia la formación permanente en Extremadura para orientar la mejora de la formación inicial, en la Orden de 25 de noviembre (2016) por la que se aprueba el Plan Marco de Formación Permanente del Profesorado de la Comunidad Autónoma de Extremadura, se puede observar que ésta se centra en cuatro ejes, relacionados con la mejora de la cualificación profesional, la mejora de las prácticas educativas, el impulso de la formación en los propios centros y el impulso de las metodologías activas, la innovación educativa y la investigación. Todas estas cuestiones se presentan a continuación en la siguiente tabla.

Tabla 1

Elementos clave en el análisis de la formación inicial desde una perspectiva extremeña

\begin{tabular}{|c|c|}
\hline Elementos clave & Fuentes \\
\hline Atraer perfiles de calidad & Valdés y Gutiérrez-Esteban (2016) \\
\hline Masificación de las aulas & Valdés y Gutiérrez-Esteban (2016) \\
\hline Relación teoría práctica & $\begin{array}{l}\text { Gutiérrez-Esteban (2011), Valdés y } \\
\text { Gutiérrez-Esteban (2016), ORDEN de } 25 \text { de } \\
\text { noviembre (2016) }\end{array}$ \\
\hline Falta de vinculación con las escuelas & Valdés y Gutiérrez-Esteban (2016) \\
\hline $\begin{array}{l}\text { Responde a las demandas actuales/Formar } \\
\text { habilidades del s. XXI }\end{array}$ & $\begin{array}{l}\text { Hernández-Rincón et al. (2012), Gutiérrez- } \\
\text { Esteban y Becerra-Traver (2014), Moreno- } \\
\text { Crespo y Moreno-Fernández (2015), } \\
\text { Valverde Berrocoso (2015), Del Rosal et al. } \\
\text { (2018) }\end{array}$ \\
\hline $\begin{array}{l}\text { Impulso de metodologías activas, innovación } \\
\text { e investigación }\end{array}$ & $\begin{array}{l}\text { Borrero y Blázquez (2018), ORDEN de } 25 \text { de } \\
\text { noviembre (2016) }\end{array}$ \\
\hline
\end{tabular}

Como síntesis de lo expuesto, resulta preciso establecer mecanismos para atraer perfiles de calidad a la formación, medida que podría contribuir a poner fin a la masificación en estos estudios universitarios, como consecuencia de la cantidad de alumnado que llega a la formación del profesorado sin tener verdaderamente clara su vocación (Sánchez Lissen, 2002).

Como elementos fundamentales, se encuentran también, el cambio que debe realizarse para otorgar un carácter más práctico a la formación y establecer lazos con los centros educativos. Por último, parece imprescindible atender a las demandas actuales que giran en torno a la introducción de metodologías activas, innovadoras y la capacitación de docentes como investigadoras e investigadores. 


\section{Ideas finales}

Con todo, resulta necesario que se produzca en las facultades de Educación y de formación del profesorado una capacitación que sirva para responder a los retos sociales que se pueden observar al hacer un análisis de la realidad social y que están estrechamente vinculados al ámbito educativo. Castillo y Gamboa (2012) recogen muchos de ellos, como son el fracaso escolar, la desigualdad entre zonas rurales y urbanas, la desmotivación, la convivencia en ambientes multiculturales, la desactualización del currículum educativo, la inequidad de género, la capacidad para analizar el entorno social o una interpretación crítica de los medios de comunicación social. En definitiva, no se debe seguir enmarcando la formación inicial del profesorado en contextos idealizados o estandarizados, sino prepararles para la realidad y la diversidad que van a encontrarse en las aulas.

Para ello, es preciso alejarnos de enfoques formativos teórico-prácticos y acercarnos, como propone Díaz Quero (2004), a un enfoque práctico-práctico que permita al profesorado en formación poner en marcha y de manera tangible sus ideas. Se trata de partir de experiencias de aprendizaje, como base para una formación en innovación, como apuntan Margalef y Álvarez Méndez (2005), tratando de perder el miedo a la innovación, dando paso a la creación y aprendiendo a poner en marcha nuevas ideas y proyectos.

En la misma línea, Reis-Jorge et al. (2020) y Trujillo et al. (2020) alertan de la necesidad de una formación de perfiles de profesorado investigador, con las competencias necesarias para afrontar los procesos de innovación educativa y los desafíos que implica la complejidad de la nueva realidad educativa. Esta formación refuerza la autonomía del profesorado y lo habilita para incidir en el contexto de la práctica docente a través de procesos de investigación y reflexión.

En consonancia a todo ello se apunta, entre otras propuestas, a la formación de un profesorado "artivista" (Mesías-Lema, 2018). Esto supone incluir dentro de la formación del profesorado, aquellos procesos subjetivos y personales que influyen en la construcción de la identidad docente que ya se han mencionado en este apartado. Lo que conlleva avivar entre el profesorado en formación inicial los retos educativos y sociales sobre los que el futuro profesorado podrá hacer activismo desde los centros docentes. Esto implica, en definitiva, formarles para que los procesos de aprendizaje que emprendan en las aulas posean un cariz ético, político, creativo y cultural, sensible al entorno del centro educativo y al contexto global. Significa, por tanto, apostar por el desarrollo de un perfil de profesorado apasionado, implicado y optimista. Capaz de intervenir dentro de la realidad educativa y no al margen de ella, siendo capaz también de conectar con el alumnado, propiciando ambientes de confianza que son clave para que éste dé pasos adelante en los procesos de aprendizaje, todo ello despertando y alentando un pensamiento crítico que promueva la justicia social entre la ciudadanía (Mesías-Lema, 2018).

Desde otro enfoque, basado en la diversidad y la igualdad en las aulas, Sales (2010) propone seis principios a tener en cuenta dentro de la formación del profesorado: entender la capacitación desde un enfoque más amplio de transformación social y cultural favoreciendo el pensamiento crítico; fomentar prácticas que permitan la democratización de los centros educativos a través de la cultura de participación; favorecer una educación dialógica que posibilite una comunicación efectiva entre los diferentes agentes de la comunidad educativa; adquirir una perspectiva sensible a las realidades de los diferentes grupos sociales y culturales; permitir, desde la pedagogía inclusiva e intercultural, que el alumnado sea capaz de construir conocimiento por sí mismo, con una actitud crítica y reflexiva de la realidad y transmitir el compromiso deseable del profesorado con el cambio social.

Mientras tanto, Santana y Bogoya (2007) y Blanchard y Fernandes Procópio (2021) resaltan la interrelación manifiesta entre profesorado y realidad social, que envuelve a los centros educativos. Por consiguiente, se precisa una formación inicial del profesorado de vanguardia, en relación con los cambios sociales, económicos y políticos, de manera que capacite al futuro profesorado como agentes sociales a través de procesos reflexivos que posibiliten la construcción y ensamblaje de las características necesarias para formar docentes contemporáneos, capaces de vencer el anacronismo que impera en la actualidad. En consonancia, Carmona (2008) aboga por una formación filosófica del profesorado, de carácter humanista, como también sugieren Pallarès (2020) y Carabantes y Contreras Salinas (2020), que fortalezca la preparación ética, en valores y reflexiva que despierte el pensamiento crítico del profesorado en contraposición a una formación tecnócrata, meramente instrumental que no es capaz de dar respuesta a las necesidades del presente y el futuro.

\section{Referencias}

Acosta, W. (2018). Innovación social educativa: una metodología de innovación 3.0 para la educación. Revista de la Universidad de La Salle, 75, 39-53. 
Adell, J. y Castañeda, L. (2012). Tecnologías emergentes, ¿pedagogías emergentes?. En J. Hernández, M. Pennesi, D. Sobrino y A. Vázquez (coord.). Tendencias emergentes en educación con TIC (págs. 13-32). Asociación Espiral, Educación y Tecnología.

Aguerrondo, I. (1999). El nuevo paradigma de la educación para el siglo XXI. Organización de Estados Iberoamericanos para la Educación, la Ciencia y la Cultura.

Alférez, A., y Comas, M. À. (2015). La innovación didáctica en la formación inicial del profesorado mediante la implementación de la Responsabilidad Social curricular. REDU. Revista de Docencia Universitaria, 13(3), 255-274.

Álvarez Álvarez, C. (2015). De la innovación educativa a la transformación social: teoría y práctica. Intangible Capital, 11(3), 285-292. http://dx.doi.org/10.3926/ic.663

Barraza, A., Cárdenas, T. D. J., y Hernández, C. (2013). ¿Cómo elaborar proyectos de innovación educativa?. Universidad Pedagógica de Durango.

Blanchard, M., y Fernandes Procópio, L. F. (2021). Claves y proceso para configurar la identidad del 'docente educador' desde la formación inicial. Acta Scientiarum. Education, 43, 1-13. http://dx.doi.org/10.4025/actascieduc.v43i1.56997

Blanco, M. T., y Bonell, R. (2019). Cómo la educación puede cambiar el mundo y cómo el mundo puede cambiar la educación. En Larragueta, M. y Ceballos, I. (A.), Educación y transformación social y cultural (pp. 101-123). Universitas Editorial.

Blas García, J. (2018). Innovación social educativa. Aula de innovación educativa, 277, 10.

Borrero, R., y Blázquez, F. (2018) La Interculturalidad en Extremadura: tareas pendientes para una escuela inclusiva del s. XXI. Educatio Siglo XXI, 36(2), 65-92. https://doi.org/10.6018/i/333121

Carabantes, E., y Contreras-Salinas, S. (2020). Educación humanizada para una democracia humanamente democrática. Revista Educación, 44(1), 584-597. https://doi.org/10.15517/revedu.v44i1.37637

Carbonell, J. (2002). El profesorado y la innovación educativa. En P. Cañal de León, (Coord), La Innovación Educativa (pp. 11-26). Akal.

Carmona, M. (2008). Hacia una formación docente reflexiva y crítica: fundamentos filosóficos. Revista de teoría y didáctica de las ciencias sociales, 13, 125-146.

Castillo, M., y Gamboa, R. (2012). Desafíos de la educación en la sociedad actual. Diálogos educativos, (24), 5569.

Cieza, J. A. (2006). Educación comunitaria. Revista de educación, 339, 765-799.

Cisneros Quintanilla, P. F., y Mendoza Bravo, K. L. (2018). Vinculación Universidad-Sociedad: espacio para generar creatividad e innovación. Killkeana sociales: Revista de Investigación Científica, 2(2), 53-58. https://doi.org/10.26871/killkana social.v2i2.304

Contreras, J., y Pérez de Lara, N. (2010). Investigar la experiencia educativa. Morata.

Cotte, A., y Gámez, J. A. (2012). Hacia una escuela de pensamiento en gestión e innovación social. Revista de la Universidad de La Salle, 58, 143-181.

Del Rosal, I., Moreno-Manso, J. M., y Bermejo, M. L. (2018). Inteligencia emocional y rendimiento académico en futuros maestros de la Universidad de Extremadura. Profesorado, revista de currículum y formación del profesorado, 22(1), 257-275.

Díaz Quero, V. (2004). Teoría emergente en la construcción del saber pedagógico. Telos: Revista de Estudios Interdisciplinarios en Ciencias Sociales, 6(2), 169-193.

Domínguez Pachón, M. J. (2009). Responsabilidad social universitaria. Humanismo y trabajo social, 8, 37-67.

Echevarría, J. (2008). El manual de Oslo y la innovación social. Arbor, 184(732), 609-618.

Fullan, M. (2002). Las fuerzas del cambio. Explorando las profundidades de la reforma educativa. Akal.

García Ramos, J. M., De la Calle, C., Valbuena, M. C., y De Dios, T. (2016). Hacia la validación del constructo responsabilidad social del estudiante universitario (RSEU). Bordón. Revista de pedagogía, 68(3), 41- 58. http://dx.doi.org/10.13042/Bordon.2016.68303

Garnica, E. y Franco, J. A. (2020). Gestión de la innovación en las instituciones de educación superior. Signos, Investigación en sistemas de gestion, 13(1), 16-25. https://doi.org/10.15332/24631140.6338

Gros, B. (2015). La caída de los muros del conocimiento en la sociedad digital y las pedagogías emergentes. Education in the knowledge society, 16(1), 58-68. https://doi.org/10.14201/eks20151615868

Grosso, J. L. (2018). Universidad, historia e innovación: territorios críticos. Revista de Divulgación de Experiencias Pedagógicas, 8, 44-49.

Gutiérrez-Esteban P. (2011). La formación del profesorado en Extremadura: evolución y perspectiva. Revista de estudios extremeños, 67(3), 1227-1260.

Gutiérrez-Esteban, P., y Becerra-Traver, M. T. (2014). Los Entornos Personales de Aprendizaje (PLE). Una experiencia de aprendizaje informal en la formación inicial del profesorado. RELATEC: Revista Latinoamericana de Tecnología Educativa, 13(2), 49-60. 
Habermas, J. (1982). Conocimiento e Interés. Taurus.

Hernández-Rincón, M. L., Gutiérrez-Esteban, P., y Yuste, R. (2012). Competencias TIC de futuros maestros y maestras de Educación Infantil y Primaria. ¿Diferentes o similares en el día a día y en la profesión docente?. En Las competencias básicas: competencias profesionales del docente (pp. 999-1008). Ediciones de la Universidad de Castilla-La Mancha.

Huertas, P. A. (2013). Aprhenciones abiertas sobre el retorno de la educación a su elemento humanista. Nodos y Nudos, 4(35), 108-112.

Lampert, E. (2003). Educación: visión panorámica mundial y perspectivas para el siglo XXI. Perfiles Educativos, 25(101), 7-22.

Leite, B., y Beltrán, J. (2012). Universidad y sociedad: la pertinencia de educación superior para una ciudadanía plena. Revista Lusófona de Educação, 21, 33-52.

Margalef, L., y Álvarez Méndez, J. M. (2005). La formación del profesorado universitario para la innovación en el marco de la integración del Espacio Europeo de Educación Superior. Revista de educación, 337, 51 70.

Mesías-Lema, J. M. (2018). Artivismo y compromiso social: Transformar la formación del profesorado desde la sensibilidad. Comunicar: Revista cientifica iberoamericana de comunicación y educación, 57, 19-28. https://doi.org/10.3916/C57-2018-02

Moreno-Crespo, P., y Moreno-Fernández, O. (2015). Problemas socioambientales: concepciones del profesorado en formación inicial. Andamios, 12(29), 73-96.

Navas, M. E. y Romero, J. (2016). Responsabilidad social universitaria: impactos de la universidad libre, sede Cartagena, en su gestión socialmente responsable. Saber, Ciencia y Libertad, 11(1), 187-196.

Novoa, A., y Camacho, C. A. (2017). Innovación y comunitarización en la educación. Revista De La Universidad De La Salle, 73, 13-31.

Orden de 25 de noviembre de 2016 por la que se aprueba el Plan Marco de Formación Permanente del Profesorado de la Comunidad Autónoma de Extremadura. Diario Oficial de Extremadura, 230, de 30 de noviembre de 2016, 31187-31197. https://bit.ly/3FIYriN

Palacios-Acosta, O. D., y Sainz-Ucros, S. M. (2019). Desde la educación humanista, hasta la escuela humanitaria. Polo del Conocimiento: Revista cientifico-profesional, 4(3), 294-317. https://doi.org/10.23857/pc.v4i3.947

Pallarès, M. (2020). Educación humanizada. Una aproximación a partir del legado de Heinrich Rombach. Estudios sobre Educación, 38, 9-27. https://doi.org/10.15581/004.38.9-27

Roa, J. D. (2017) La Innovación Social Educativa (ISE) como herramienta metodológica para la búsqueda de una educación con sentido. Rev. Guillermo de Ockham, 15(1), 109-117.

Sancho Gil, J. M. (2010). ¿En qué dirección (es) se orientará la Investigación sobre cambio educativo en los próximos diez años? La opinión de los especialistas. Revista mexicana de investigación educativa, 15(47), 1093-1145.

Santana, C., y Bogoya, N. (2007). ¿Cuáles son los retos para el docente contemporáneo?. Nodos y Nudos, $3(22), 4-13$.

Tomé, A. (2019). La educación feminista contra el sexismo educativo y cultural. Dosier Graó, 4, 8-14.

Turrado, M. Á. (2015). Innovaciones educativas en los centros de Educación Infantil y Primaria de la provincia de León (Tesis de Doctorado, Universidad de León).

Reis-Jorge, J., Ferreira, M., y Olcina, G. (2020). La figura del profesorado-investigador en la reconstrucción de la profesionalidad docente en un mundo en transformación. Revista Educación, 44(1), 509-519. http://dx.doi.org/10.15517/REVEDU.V44I1.39044

Sales, M.A. (2010). La formación intercultural inclusiva del profesorado: Hacia la transformación social. Revista Latinoamericana de Inclusión Educativa, 4(1) 65-82.

Sales, A., Traver, J. A., y Moliner, O. (2019). Redefiniendo el territorio de la escuela: espacios educativos y curriculum escolar para la transformación social. Revista Fuentes, 21(2), 177-188. http://dx.doi.org/10.12795/revistafuentes.2019.v21.i2.03

Sánchez Lissen, E. (2002). Elegir magisterio: entre la motivación, la vocación y la obligación. Escuela abierta: revista de investigación educativa, 5, 99-120.

Sancho Gil, J. M., y Correa Gorospe, J. M. (2010). Cambio y continuidad en sistemas educativos en transformación Change continuity in changing education systems. Consejo de dirección/managing board, 352, 17-21.

Serna Alcántara, G. A. (2007). Misión social y modelos de extensión universitaria: del entusiasmo al desdén. Revista Iberoamericana de Educación, 43, 3-25.

Schröder, A., \& Krüger, D. (2019). Social Innovation as a Driver for New Educational Practices: Modernising, Repairing and Transforming the Education System. Sustainability, 11(4), 1-25. 
Sureda, M. (2017). La innovación social: retos de futuro en el sector no lucrativo. Cuadernos 35, 6-7.

Surikova, S., Oganisjana, K. \& Grinberga-Zalite, G. (2015). The Role of Education in Promoting Social Innovation Processes in the Society. SOCIETY, INTEGRATION, EDUCATION. Proceedings of the International Scientific Conference, 4. 233. http://dx.doi.org/10.17770/sie2015vol4.337

Torres Pedreros, J. A. (2016). Innovación social: nuevos retos para las facultades de educación. En Educación Pedagogía e Innovación Social Educativa. (pp. 119-146). Editorial REDIPE.

Trujillo, F. T., Robles, A. S., Vázquez, A. G., Casas, E. C., Ruda, J. G., Mártínez, M. B., ... y Herrero, J. F. Á. (2020). ¿Qué podemos aprender de la escuela innovadora para la formación inicial y permanente del profesorado? Propuesta de investigación. Márgenes: Revista de Educación de la Universidad de Málaga, 1(1), 129-142. http://dx.doi.org/10.24310/mgnmar.v1i1.6637

UNESCO (1998). La Educación Superior en el Siglo XXI: Visión y Acción. Conferencia Mundial sobre la Educación Superior.

Valdés, V., y Gutiérrez Esteban, P. (2016). Desafíos en la formación inicial del profesorado. Un análisis desde las voces de sus protagonistas. Revista Internacional de Formação de Professores, 1(3), 45-58.

Valverde Berrocoso, J. (2015). La formación inicial del profesorado en el Grado en Educación Primaria. Una valoración cualitativa del diseño y desarrollo curricular de la asignatura "Recursos tecnológicos didácticos y de investigación”. Tendencias Pedagógicas, 25, 207-227.

Villa, A. (2014). La innovación social en el ámbito universitario: una propuesta para su diagnóstico y desarrollo. Revista Argentina de Educación Superior, 8, 188-218.

Villa, A. (2020). Aprendizaje Basado en Competencias: desarrollo e implantación en el ámbito universitario. REDU. Revista de Docencia Universitaria, 18(1), 19-46. 